EUROPEAN JOURNAL OF PURE AND APPLIED MATHEMATICS

Vol. 13, No. 5, 2020, 1149-1161

ISSN 1307-5543 - www.ejpam.com

Published by New York Business Global

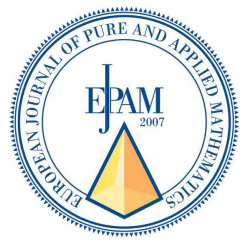

Special Issue Dedicated to

Professor Hari M. Srivastava

On the Occasion of his 80th Birthday

\title{
Computing Discrete Adriatic Indices of Probabilistic Neural Network
}

T. Deepika ${ }^{1, *}$, V. Lokesha ${ }^{2}$

1 Department of Mathematics, Dayananda Sagar University, Bengaluru, Karnataka, India.

2 Department of Mathematics, Vijayanagara Sri Krishnadevaraya University, Ballari, Karnataka, India.

\begin{abstract}
A Topological index is a numeric quantity which characterizes the whole structure of a graph. Adriatic indices are also part of topological indices, mainly it is classified into two namely extended variables and discrete adriatic indices, especially, discrete adriatic indices are analyzed on the testing sets provided by the International Academy of Mathematical Chemistry (IAMC) and it has been shown that they have good presaging substances in many compacts. This contrived attention to compute some discrete adriatic indices of probabilistic neural network.
\end{abstract}

2020 Mathematics Subject Classifications: 05C09,05C30

Key Words and Phrases: Topological indices, probabilistic neural network, degree vertex.

\section{Prologue and Provocation}

In this work peculiar attention is compensated to Adriatic indices that have been defined by D. Vukičević and M. Gasperov. Discrete Adriatic indices are the family of 148 bond-additive topological indices defined as follows.

$$
\operatorname{Adr}(G)=\sum_{u v \in E(G)} \gamma_{j}\left(\varphi_{i, a}\left(t_{u}\right), \varphi_{i, a}\left(t_{v}\right)\right)
$$

where the variables and functions can take the following values:

* Corresponding author.

DOI: https://doi.org/10.29020/nybg.ejpam.v13i5.3712

Email addresses: v.lokesha@gmail.com (V. Lokesha), sastry.deepi@gmail.com (T. Deepika) 
$\mathrm{t}_{u} \in\left\{d_{u}, D_{u}\right\} i \in\{1,2,3\} j \in\{1,2, \ldots . ., 8\}$

$\mathrm{a}= \begin{cases}\left(-1,-\frac{1}{2}, \frac{1}{2}, 1,2\right) & \text { if } i=2 \text { and } j \in(1,2, \ldots 5) \\ \left(\frac{1}{2}, 1,2\right) & \text { otherwise and } i \in(1,2) \\ \left(\frac{1}{2}, 2\right) & \text { if } i=3\end{cases}$

$$
\varphi_{1, a}(x)=\log ^{a}(x)(a>0) ; \quad \varphi_{2, a}(x)=x^{a}(a \in \mathbb{R} \backslash 0) ; \quad \varphi_{3, a}(x)=a^{x}(a>0) ;
$$

Now, the naming convention for the discrete adriatic indices is as follows:

1. $\gamma_{1}$ corresponds to Randi type; $\quad \gamma_{1}(x, y)=x . y$

- Randi type lodeg index: $\operatorname{Adr}(G)=\sum_{u v \in E(G)} \gamma_{1}\left(\varphi_{1,1}\left(d_{u}\right), \varphi_{1,1}\left(d_{v}\right)\right)$

- Randi type sdi index: $A d r(G)=\sum_{u v \in E(G)} \gamma_{1}\left(\varphi_{2,2}\left(D_{u}\right), \varphi_{2,2}\left(D_{v}\right)\right)$

- Randi type hadi index: $A d r(G)=\sum_{u v \in E(G)} \gamma_{1}\left(\varphi_{3,1 / 2}\left(D_{u}\right), \varphi_{3,1 / 2}\left(D_{v}\right)\right)$

2. $\gamma_{2}$ corresponds to sum; $\gamma_{2}(x, y)=x+y$

- sum lordeg index: $A d r(G)=\sum_{u v \in E(G)} \gamma_{2}\left(\varphi_{1,1 / 2}\left(d_{u}\right), \varphi_{1,1 / 2}\left(d_{v}\right)\right)$

3. $\gamma_{3}$ corresponds to inverse sum;

$$
\gamma_{3}(x, y)= \begin{cases}\frac{1}{x+y}, & \text { if } x+y \neq 0 \\ 0, & \text { otherwise }\end{cases}
$$

- inverse sum lordeg index: $A d r(G)=\sum_{u v \in E(G)} \gamma_{3}\left(\varphi_{1,1 / 2}\left(d_{u}\right), \varphi_{1,1 / 2}\left(d_{v}\right)\right)$

- inverse sum indeg index: $A d r(G)=\sum_{u v \in E(G)} \gamma_{3}\left(\varphi_{2,-1}\left(d_{u}\right), \varphi_{2,-1}\left(d_{v}\right)\right)$

4. $\gamma_{4}$ corresponds to misbalance; $\gamma_{4}(x, y)=|x-y|$

- misbalance lodeg index: $A d r(G)=\sum_{u v \in E(G)} \gamma_{4}\left(\varphi_{1,1}\left(d_{u}\right), \varphi_{1,1}\left(d_{v}\right)\right)$ 
- misbalance losdeg index: $A d r(G)=\sum_{u v \in E(G)} \gamma_{4}\left(\varphi_{1,2}\left(d_{u}\right), \varphi_{1,2}\left(d_{v}\right)\right)$

- misbalance rodeg index: $A d r(G)=\sum_{u v \in E(G)} \gamma_{4}\left(\varphi_{2,1 / 2}\left(d_{u}\right), \varphi_{2,1 / 2}\left(d_{v}\right)\right)$

- misbalance deg index: $A d r(G)=\sum_{u v \in E(G)} \gamma_{4}\left(\varphi_{2,1}\left(d_{u}\right), \varphi_{2,1}\left(d_{v}\right)\right)$

- misbalance hadeg index: $A d r(G)=\sum_{u v \in E(G)} \gamma_{4}\left(\varphi_{3,1 / 2}\left(d_{u}\right), \varphi_{3,1 / 2}\left(d_{v}\right)\right)$

5. $\gamma_{5}$ corresponds to inverse misbalance;

$$
\gamma_{5}(x, y)= \begin{cases}\frac{1}{|x-y|}, & \text { if } x \neq y \\ 0, & \text { if } x=y\end{cases}
$$

- misbalance indeg index: $A d r(G)=\sum_{u v \in E(G)} \gamma_{5}\left(\varphi_{2,-1}\left(d_{u}\right), \varphi_{2,-1}\left(d_{v}\right)\right)$

- misbalance irdeg index: $A d r(G)=\sum_{u v \in E(G)} \gamma_{5}\left(\varphi_{2,-1 / 2}\left(d_{u}\right), \varphi_{2,-1 / 2}\left(d_{v}\right)\right)$

- misbalance indi index: $A d r(G)=\sum_{u v \in E(G)} \gamma_{5}\left(\varphi_{2,-1}\left(D_{u}\right), \varphi_{2,-1}\left(D_{v}\right)\right)$

6. $\gamma_{6}$ corresponds to min-max;

$$
\gamma_{6}(x, y)= \begin{cases}\frac{\min \{x, y\}}{\max \{x, y\}}, & \text { if } \max \{x, y\} \neq 0 \\ 0, & \text { if } \max \{x, y\}=0\end{cases}
$$

- min-max rodeg index: $A d r(G)=\sum_{u v \in E(G)} \gamma_{6}\left(\varphi_{2,1 / 2}\left(d_{u}\right), \varphi_{2,1 / 2}\left(d_{v}\right)\right)$

- min-max sdi index: $A d r(G)=\sum_{u v \in E(G)} \gamma_{6}\left(\varphi_{2,2}\left(D_{u}\right), \varphi_{2,2}\left(D_{v}\right)\right)$

7. $\gamma_{7}$ corresponds to max-min;

$$
\gamma_{7}(x, y)= \begin{cases}\frac{\max \{x, y\}}{\min \{x, y\}}, & \text { if } \min \{x, y\} \neq 0 \\ 0, & \text { if } \min \{x, y\}=0\end{cases}
$$

- max-min rodeg index: $A d r(G)=\sum_{u v \in E(G)} \gamma_{7}\left(\varphi_{2,1 / 2}\left(d_{u}\right), \varphi_{2,1 / 2}\left(d_{v}\right)\right)$ 
- max-min deg index: $A d r(G)=\sum_{u v \in E(G)} \gamma_{7}\left(\varphi_{2,1}\left(d_{u}\right), \varphi_{2,1}\left(d_{v}\right)\right)$

- max-min sdeg index: $A d r(G)=\sum_{u v \in E(G)} \gamma_{7}\left(\varphi_{2,2}\left(d_{u}\right), \varphi_{2,2}\left(d_{v}\right)\right)$

8. $\gamma_{8}$ corresponds to symmetric division;

$$
\gamma_{8}(x, y)= \begin{cases}\frac{x}{y}+\frac{y}{x}, & \text { if } x, y \neq 0 \\ 0, & \text { otherwise }\end{cases}
$$

- symmetric division deg index: $\operatorname{Adr}(G)=\sum_{u v \in E(G)} \gamma_{8}\left(\varphi_{2,1}\left(d_{u}\right), \varphi_{2,1}\left(d_{v}\right)\right)$

The Probabilistic Neural Network $(P N N)$ gives new directions in the Quantitative Structure-Activity Relationship (QSAR)/Quantitative Structure-Property Relationship (QSPR) studies [3]. The $P N N$ methodology is applicable to classification problems and the basic underlying theory behind these probability-based methods is presented along with applications of the $P N N$ methodology.

The $P N N$ model presented identifies molecules as potential soluble epoxide hydrolase inhibitors using a binary classification scheme. This network inputs consist of a small set of descriptors that encode structural features at the molecular level (refer [4, 14]).

The topological indices are exploited to hypothesis the physical features related to the bio-activities, chemical reactivities in certain networks. In this article, we established the degree-based discrete adriatic indices of the probabilistic neural network.

Recently, $[1,12]$ exposed the 148 adriatic indices among which Symmetric division deg $(S D D)$ index is one of the discrete adriatic indices, and it has been proved as a good predictor for total surface area for polychlorobiphenyls. Newly, Lokesha et. al., $[5,6,8]$ worked out for $S D D$ index of unicyclic and bicyclic later it is also extended to tricyclic and tetracyclic, also they did for graph operations.

Newly, Deepika et al [9], computed the graph structure of $2 D$-Lattice, nanotube and nanotorus exploiting the definitions of $S D D$ and other topological indices via certain graph operators which gives more attraction towards to this index (also see $[2,10,13]$ ).

In the last decade the probabilistic neural networks are widely studied in different classification problems. These are applied to solve the problems related to email security enhancement and in the intrusion detection systems. The probabilistic neural networks are also applied in medicine such as for detecting resistivity to antibiotics, for diagnosing hepatitis, and for the quantification and segmentation of brain tissues from MR images (also see $[7,11])$. In the present study, we compute the topological indices such as newly defined adriatic indices to continue the progressive study of the probabilistic neural networks. 
The construction of the probabilistic neural network. we consider the probabilistic neural network consisting on three layers of nodes.

- The first layer called by input layer has a certain number of nodes, the second layer called by hidden layer consists on a certain number of classes such that each class contains a particular number of nodes, and the third layer called by output layer has a number of nodes equal to the number of classes of the second layer.

- The architecture of a probabilistic neural network, each node of input layer is connected to all the nodes of each class of the hidden layer and all the nodes of each class of the hidden layer are connected to a unique node of the output layer.

- Assume the input layer has $n$ nodes, the hidden layer consists on $k$ classes such that each class has $m$ nodes, and the third layer called by output layer has $k$ nodes.

Thus, a probabilistic neural network denoted by $\operatorname{PNN}(\mathrm{n}, \mathrm{k}, \mathrm{m})$ is such that $|V(P N N(n, k, m))|$ $=v=n+k(m+1)$ and $|E(P N N(n, k, m))|=e=k m(n+1)$, where $(n, k, m) \in N$ (set of natural numbers). In Fig. 1, the probabilistic neural network is shown for $n=4, k=2$ and $m=3$.

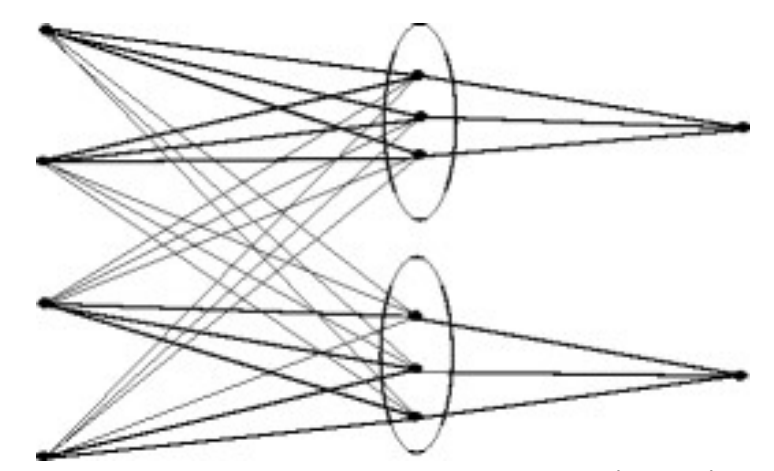

Figure 1: The probabilistic neural network $\operatorname{PNN}(4,2,3)$

We define the partitions of the edge set of $P N N(n, k, m)$ with respect to degree of vertices. There are two types of edges with respect to degrees of end vertices in $P N N(n, k, m)$, namely with degrees of end vertices $k m, n+1$ and degrees of end vertices $n+1, m$. Thus, we have shown in the following table 1.

Table 1: The edge partition of the edges of $P N N(n, k, m)$ based on degrees of end vertices

\begin{tabular}{|c|c|c|}
\hline$E_{\{d(u), d(v)\}}$ & $E_{k m, n+1}$ & $E_{n+1, m}$ \\
\hline$E_{\{d(u), d(v)\}}$ & $n k m$ & $k m$ \\
\hline
\end{tabular}

\section{Main Results}

In this section, we established some results for probabilistic neural networks using different adriatic indices 
Theorem 1. Let $G$ be the probabilistic neural network $P N N(n, k, m)$ then for $(n, k, m) \geq$ 1 , its misbalance deg index, misbalance indeg index, misbalance rodeg index and misbalance irdeg index is given by

$$
M B I_{\alpha}(G)=\left\{\begin{array}{l}
n k m|k m-(n+1)|+k m|(n+1)-m| \text { for } \alpha=1 \\
n k m\left|\frac{1}{k m}-\frac{1}{n+1}\right|+k m\left|\frac{1}{n+1}-\frac{1}{m}\right| \text { for } \alpha=-1 \\
n k m|\sqrt{k m}-\sqrt{n+1}|+k m|\sqrt{n+1}-\sqrt{m}| \text { for } \alpha=\frac{1}{2} \\
n k m\left|\frac{1}{\sqrt{k m}}-\frac{1}{\sqrt{n+1}}\right|+k m\left|\frac{1}{\sqrt{n+1}}-\frac{1}{\sqrt{m}}\right| \text { for } \alpha=-\frac{1}{2}
\end{array}\right.
$$

Proof. Let $G$ be the $P N N(n, k, m)$, for $(n, k, m) \geq 1$. The number of vertices and edges in $P N N(n, k, m)$ are $n+k(m+1)$ and $k m(n+1)$ respectively. There are two types of edges in $P N N(n, k, m)$ based on degrees of end vertices of each edge. Table 1 shows such an edge partition of $P N N(n, k, m)$. Now by using the definition of Misbalance indices and table 1 we obtain the required results as follows

We consider the following cases for the possible values of $\alpha$

Case $1: \alpha=1$

Applying the formula of Misbalance deg index for $\alpha=1$ and by using edge partition given in table 1 we get

$$
\begin{aligned}
M B I_{1} & =\sum_{u v \in E(G)}\left|d_{u}-d_{v}\right| \\
& =\sum_{u v \in E_{k m, n+1}}\left|d_{u}-d_{v}\right|+\sum_{u v \in E_{n+1, m}}\left|d_{u}-d_{v}\right| \\
& =n k m|k m-(n+1)|+k m|(n+1)-m| \\
& =k m[n|k m-(n+1)|+|(n+1)-m|]
\end{aligned}
$$

Case 2 : $\alpha=-1$, Applying the formula of Misbalance indeg index for $\alpha=-1$ and by using edge partition given in table 1 we get

$$
\begin{aligned}
M B I_{-1} & =\sum_{u v \in E(G)}\left|\frac{1}{d_{u}}-\frac{1}{d_{v}}\right| \\
& =\sum_{u v \in E_{k m, n+1}}\left|\frac{1}{d_{u}}-\frac{1}{d_{v}}\right|+\sum_{u v \in E_{n+1, m}}\left|\frac{1}{d_{u}}-\frac{1}{d_{v}}\right| \\
& =n k m\left|\frac{1}{k m}-\frac{1}{n+1}\right|+k m\left|\frac{1}{n+1}-\frac{1}{m}\right| \\
& =k m\left[n\left|\frac{1}{k m}-\frac{1}{n+1}\right|+\left|\frac{1}{n+1}-\frac{1}{m}\right|\right]
\end{aligned}
$$


Case $3: \alpha=\frac{1}{2}$, Edge partition for Misbalance rodeg index when $\alpha=\frac{1}{2}$ we get

$$
\begin{aligned}
M B I_{\frac{1}{2}} & =\sum_{u v \in E(G)}\left|\sqrt{d_{u}}-\sqrt{d_{v}}\right| \\
& =\sum_{u v \in E_{k m, n+1}}\left|\sqrt{d_{u}}-\sqrt{d_{v}}\right|+\sum_{u v \in E_{n+1, m}}\left|\sqrt{d_{u}}-\sqrt{d_{v}}\right| \\
& =n k m|\sqrt{k m}-\sqrt{n+1}|+k m|\sqrt{n+1}-\sqrt{m}| \\
& =[|\sqrt{k m}-\sqrt{n+1}|+|\sqrt{n+1}-\sqrt{m}|]
\end{aligned}
$$

Case $4: \alpha=-\frac{1}{2}$, For Misbalance irdeg index when $\alpha=-\frac{1}{2}$ using edge partition given in table 1 we get

$$
\begin{aligned}
M B I_{-\frac{1}{2}} & =\sum_{u v \in E(G)}\left|\frac{1}{\sqrt{d_{u}}}-\frac{1}{\sqrt{d_{v}}}\right| \\
& =\sum_{u v \in E_{k m, n+1}}\left|\frac{1}{\sqrt{d_{u}}}-\frac{1}{\sqrt{d_{v}}}\right|+\sum_{u v \in E_{n+1, m}}\left|\frac{1}{\sqrt{d_{u}}}-\frac{1}{\sqrt{d_{v}}}\right| \\
& =n k m\left|\frac{1}{\sqrt{k m}}-\frac{1}{\sqrt{n+1}}\right|+k m\left|\frac{1}{\sqrt{n+1}}-\frac{1}{\sqrt{m}}\right| \\
& =k m\left[n\left|\frac{1}{\sqrt{k m}}-\frac{1}{\sqrt{n+1}}\right|+\left|\frac{1}{\sqrt{n+1}}-\frac{1}{\sqrt{m}}\right|\right]
\end{aligned}
$$

Theorem 2. Let $G$ be the probabilistic neural network $P N N(n, k, m)$ then for $(n, k, m) \geq$ 1, its max-min deg index, max-min sdeg index and max rodeg index is given by

$$
\begin{aligned}
\text { Max-min deg index } & =\frac{k}{n+1}\left(m^{2} n k+(n+1)^{2}\right) \\
\text { Max-min sdeg index } & =k\left[\frac{k^{2} m^{4} n+(n+1)^{4}}{(n+1)^{2} m}\right] \\
\text { Max-rodeg index } & =k m\left[n \sqrt{\frac{k m}{n+1}}+\sqrt{\frac{n+1}{m}}\right]
\end{aligned}
$$

Proof. Let $G$ be the $P N N(n, k, m)$, for $(n, k, m) \geq 1$. The number of vertices and edges in $P N N(n, k, m)$ are $n+k(m+1)$ and $k m(n+1)$ respectively. There are two types of edges in $P N N(n, k, m)$ based on degrees of end vertices of each edge. Table 1 shows such an edge partition of $\operatorname{PNN}(n, k, m)$. Now by using the formulas of max-min deg index, max-min sdeg index, max rodeg index and table 1 we obtain the required results as follows

$$
\text { Max-min deg index }=\sum_{u v \in E(G)} \frac{\max \left(d_{u}, d_{v}\right)}{\min \left(d_{u}, d_{v}\right)}
$$




$$
\begin{aligned}
& =\sum_{u v \in E_{k m, n+1}} \frac{\max \left(d_{u}, d_{v}\right)}{\min \left(d_{u}, d_{v}\right)}+\sum_{u v \in E_{n+1, m}} \frac{\max \left(d_{u}, d_{v}\right)}{\min \left(d_{u}, d_{v}\right)} \\
& =n k m\left(\frac{k m}{n+1}\right)+k m\left(\frac{n+1}{m}\right) \\
& =\frac{k}{n+1}\left(m^{2} n k+(n+1)^{2}\right)
\end{aligned}
$$

$$
\begin{aligned}
\text { Max-min sdeg index } & =\sum_{u v \in E(G)}\left(\frac{\max \left(d_{u}, d_{v}\right)}{\min \left(d_{u}, d_{v}\right)}\right)^{2} \\
& =\sum_{u v \in E_{k m, n+1}}\left(\frac{\max \left(d_{u}, d_{v}\right)}{\min \left(d_{u}, d_{v}\right)}\right)^{2}+\sum_{u v \in E_{n+1, m}}\left(\frac{\max \left(d_{u}, d_{v}\right)}{\min \left(d_{u}, d_{v}\right)}\right)^{2} \\
& =n k m\left(\frac{k m}{n+1}\right)^{2}+k m\left(\frac{n+1}{m}\right)^{2} \\
& =k\left[\frac{k^{2} m^{4} n+(n+1)^{4}}{(n+1)^{2} m}\right]
\end{aligned}
$$$$
\text { Max rodeg index }=\sum_{u v \in E(G)} \sqrt{\frac{\max \left(d_{u}, d_{v}\right)}{\min \left(d_{u}, d_{v}\right)}}
$$$$
=\sum_{u v \in E_{k m, n+1}} \sqrt{\frac{\max \left(d_{u}, d_{v}\right)}{\min \left(d_{u}, d_{v}\right)}}+\sum_{u v \in E_{n+1, m}} \sqrt{\frac{\max \left(d_{u}, d_{v}\right)}{\min \left(d_{u}, d_{v}\right)}}
$$$$
=n k m \sqrt{\frac{k m}{n+1}}+k m \sqrt{\frac{n+1}{m}}
$$$$
=k m\left[n \sqrt{\frac{k m}{n+1}}+\sqrt{\frac{n+1}{m}}\right]
$$

Theorem 3. Let $G$ be the probabilistic neural network $P N N(n, k, m)$ then for $(n, k, m) \geq$ 1, its min-max rodeg index and min-max sdi index is given by

$$
\begin{aligned}
\text { Min-max rodeg index } & =k m\left[n \sqrt{\frac{n+1}{k m}}+\sqrt{\frac{m}{n+1}}\right] \\
\text { Min-max sdi index } & =\frac{n(n+1)^{4}+k^{2} m^{4}}{k m(n+1)^{2}}
\end{aligned}
$$

Proof. Let $G$ be the $P N N(n, k, m)$, for $(n, k, m) \geq 1$. The number of vertices and edges in $P N N(n, k, m)$ are $n+k(m+1)$ and $k m(n+1)$ respectively. There are two types of edges in $P N N(n, k, m)$ based on degrees of end vertices of each edge. Table 1 shows 
such an edge partition of $P N N(n, k, m)$. Now by using the formulas of min-max rodeg index, min-max sdi index and table 1 we obtain the required results as follows

$$
\begin{aligned}
\text { Min-max rodeg index } & =\sum_{u v \in E(G)} \sqrt{\frac{\min \left(d_{u}, d_{v}\right)}{\max \left(d_{u}, d_{v}\right)}} \\
& =\sum_{u v \in E_{k m, n+1}} \sqrt{\frac{\min \left(d_{u}, d_{v}\right)}{\max \left(d_{u}, d_{v}\right)}}+\sum_{u v \in E_{n+1, m}} \sqrt{\frac{\min \left(d_{u}, d_{v}\right)}{\max \left(d_{u}, d_{v}\right)}} \\
& =n k m \sqrt{\frac{n+1}{k m}}+k m \sqrt{\frac{m}{n+1}} \\
& =k m\left[n \sqrt{\frac{n+1}{k m}}+\sqrt{\frac{m}{n+1}}\right] \\
\text { Min-max sdi index } & \sum_{u v \in E(G)}\left(\frac{\min \left(d_{u}, d_{v}\right)}{\max \left(d_{u}, d_{v}\right)}\right)^{2} \\
& =\sum_{u v \in E_{k m, n+1}}\left(\frac{\min \left(d_{u}, d_{v}\right)}{\max \left(d_{u}, d_{v}\right)}\right)^{2}+\sum_{u v \in E_{n+1, m}}\left(\frac{\min \left(d_{u}, d_{v}\right)}{\max \left(d_{u}, d_{v}\right)}\right)^{2} \\
& =\frac{n k m\left(\frac{n+1}{k m}\right)^{2}+k m\left(\frac{m}{n+1}\right)^{2}}{k(n+1)^{4}+k^{2} m^{4}} \\
& =\frac{n m(n+1)^{2}}{k(n)}
\end{aligned}
$$

Theorem 4. Let $G$ be the probabilistic neural network $P N N(n, k, m)$ then for $(n, k, m) \geq$ 1, its Inverse sum indeg index and Symmetric division deg index is given by

$$
\begin{aligned}
I S I(G) & =k m^{2}\left[\frac{k(m+n)(2 n+1)+(n+1)^{2}}{(m+n+1)(k m+n+1)}\right] \\
S D D(G) & =\frac{1}{n+1}\left[n(n+1)^{2}+k\left(m^{2}(1+k n)+n^{2}+3\right)\right]
\end{aligned}
$$

Proof. Let $G$ be the $P N N(n, k, m)$, for $(n, k, m) \geq 1$. The number of vertices and edges in $P N N(n, k, m)$ are $n+k(m+1)$ and $k m(n+1)$ respectively. There are two types of edges in $P N N(n, k, m)$ based on degrees of end vertices of each edge. Table 1 shows such an edge partition of $P N N(n, k, m)$. Now by using the formulas of $I S I, S D D$ and table 1 we obtain the required results as follows

$$
I S I(G)=\sum_{u v \in E(G)} \frac{d_{u} d_{v}}{d_{u}+d_{v}}
$$


T. Deepika, V. Lokesha / Eur. J. Pure Appl. Math, 13 (5) (2020), 1149-1161

$$
\begin{aligned}
& =\sum_{u v \in E_{k m, n+1}} \frac{d_{u} d_{v}}{d_{u}+d_{v}}+\sum_{u v \in E_{n+1, m}} \frac{d_{u} d_{v}}{d_{u}+d_{v}} \\
& =n k m\left[\frac{(k m)(n+1)}{k m+(n+1)}\right]+k m\left[\frac{(n+1)(m)}{(n+1)+m}\right. \\
& =k m\left[n\left(\frac{k m}{k m+1}\right)+\left(\frac{m}{1+m}\right)\right] \quad= \\
& k m^{2}\left[\frac{k(m+n)(2 n+1)+(n+1)^{2}}{(m+n+1)(k m+n+1)}\right] \\
& \text { Min-maxsdiindex }=\sum_{u v \in E(G)}\left(\frac{\min \left(d_{u}, d_{v}\right)}{\max \left(d_{u}, d_{v}\right)}\right)^{2} \\
& =\sum_{u v \in E_{k m, n+1}}\left(\frac{\min \left(d_{u}, d_{v}\right)}{\max \left(d_{u}, d_{v}\right)}\right)^{2}+\sum_{u v \in E_{n+1, m}}\left(\frac{\min \left(d_{u}, d_{v}\right)}{\max \left(d_{u}, d_{v}\right)}\right)^{2} \\
& =n k m\left(\frac{n+1}{k m}\right)^{2}+k m\left(\frac{m}{n+1}\right)^{2} \\
& =\frac{n(n+1)^{4}+k^{2} m^{4}}{k m(n+1)^{2}}
\end{aligned}
$$

Let $G$ be the probabilistic neural network $P N N(n, k, m)$ then for $(n, k, m) \geq 1$, its adriatic indices of PNN of $\gamma_{j}(x, y)(j=1,2, \ldots 8)$ index is given by

- $A d r(P N N)_{\gamma_{1}(x, y)}= \begin{cases}k m \cdot \log (n+1) \cdot \log \left(k^{n} m^{n+1}\right), & \text { if } \varphi_{i, a}=\varphi_{1,1} \\ 2 n^{4}+58 k^{4}+6 m^{4}+60, & \text { if } \varphi_{2,2} \\ \frac{2}{2^{2 n}}+\frac{58}{2^{2 k}}+\frac{6}{2^{2 m}}+15, & \text { if } \varphi_{3,1 / 2} .\end{cases}$

- $A d r(P N N)_{\gamma_{2}(x, y)}=k m(n+1) \sqrt{\log (n+1)}+\sqrt{\log (k m)}+\sqrt{\log (m)}$, if $\varphi_{i, a}=\varphi_{1,1 / 2}$

- $\operatorname{Adr}(P N N)_{\gamma_{3}(x, y)}= \begin{cases}\frac{1}{k m(n+1) \sqrt{\log (n+1)}+\sqrt{\log (k m)}+\sqrt{\log (m)},} & \text { if } \varphi_{i, a}=\varphi_{1,1 / 2} \\ k m^{2}\left[\frac{n k(n+1)}{k m+n+1}+\frac{n+1}{m+n+1}\right], & \text { if } \varphi_{2,-1}\end{cases}$

- $A d r(P N N)_{\gamma_{4}(x, y)}= \begin{cases}k m\left[\log \left(k^{n} m^{n-1}\right)-(n-1) \log (n+1)\right], & \text { if } \varphi_{i, a}=\varphi_{1,1} \\ 2 k m\left[\log \left(k^{n} m^{n-1}\right)-(n-1) \log (n+1)\right], & \text { if } \varphi_{1,2} \\ n k m|\sqrt{k m}-\sqrt{n+1}|+k m|\sqrt{n+1}-\sqrt{m}|, & \text { if } \varphi_{2,1 / 2} \\ n k m|k m-(n+1)|+k m|(n+1)-m|, & \text { if } \varphi_{2,1} \\ k m\left[n 2^{-k m}+2^{n+1}-n 2^{-(n+1)}-2^{-m}\right], & \text { if } \varphi_{3,1 / 2}\end{cases}$ 
- $A d r(P N N)_{\gamma_{5}(x, y)}= \begin{cases}n k m\left|\frac{1}{k m}-\frac{1}{n+1}\right|+k m\left|\frac{1}{n+1}-\frac{1}{m}\right|, & \text { if } \varphi_{i, a}=\varphi_{2,-1} \\ n k m\left|\frac{1}{\sqrt{k m}}-\frac{1}{\sqrt{n+1}}\right|+k m\left|\frac{1}{\sqrt{n+1}}-\frac{1}{\sqrt{m}}\right|, & \text { if } \varphi_{2,-1 / 2} \\ 0, & \text { if } \varphi_{2,-1}\end{cases}$

- $A d r(P N N)_{\gamma_{6}(x, y)}= \begin{cases}k m\left[n \sqrt{\frac{n+1}{k m}}+\sqrt{\frac{m}{n+1}}\right], & \text { if } \varphi_{i, a}=\varphi_{2,1 / 2} \\ 14(n+k+m), & \text { if } \varphi_{2,2}\end{cases}$

- $A d r(P N N)_{\gamma_{7}(x, y)}= \begin{cases}k m\left[n \sqrt{\frac{k m}{n+1}}+\sqrt{\frac{n+1}{m}}\right], & \text { if } \varphi_{i, a}=\varphi_{2,1 / 2} \\ \frac{k}{n+1}\left(m^{2} n k+(n+1)^{2}\right), & \text { if } \varphi_{2,1} \\ k\left[\frac{k^{2} m^{4} n+(n+1)^{4}}{(n+1)^{2} m}\right], & \text { if } \varphi_{2,2}\end{cases}$

- $A d r(P N N)_{\gamma_{8}(x, y)}=\frac{1}{n+1}\left[n(n+1)^{2}+k\left(m^{2}(1+k n)+n^{2}+3\right)\right]$, if $\varphi_{i, a}=\varphi_{2,1}$

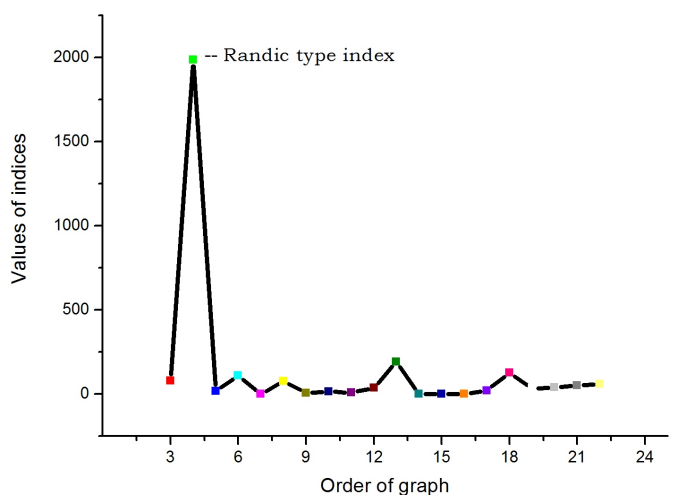

Figure 2: The probabilistic neural network $\operatorname{PNN}(n, n, 1)$

\section{Conclusion}

We compare the results of the computed adriatic indices for the probabilistic neural network $P N N(n, k, m)$ with the help of software package. In the main results the formulae of all the indices are computed in term of $n, k$, and $m$, where $n$ is number of nodes in first layer, the second layer is consisting on $k$ classes such that each class has $m$ nodes and the third layer contains $k$ nodes. Moreover, the total number of vertices in the probabilistic neural network $P N N(n, k, m)$ (order of $P N N(n, k, m)$ ) is $|V(P N N(n, k, m))|=v=$ $n+k(m+1)$. If we assume $k=n$ and $m=1$, then the probabilistic neural network becomes $P N N(n, n, 1)$ with order $v=3 n$, where $n$ is a natural number.

In Fig. 2, along the horizontal line the values of $v$ for the probabilistic neural network 
$P N N(n, n, 1)$ are taken and along the vertical line the computed values of the indices are shown. Among them the Randic type index is dominant. Moreover, all the adriatic indices remain constant approximately with increasing values of $v$. In this paper, the adriatic indices of the probabilistic neural network are studied and the analytical closed formulas are determined that will help to understand the underlying topologies related to the physical features of this network.

\section{Acknowledgements}

The authors thank the referees for their useful comments that helped to improve this paper.

\section{References}

[1] V. Alexander. Upper and lower bounds of symmetric division deg index. Iranian Journal of Mathematical Chemistry, 5(2):91-98, 2014.

[2] N. Ananda, P.S. Ranjini, V. Lokesha, and S.A. Wazzan. Subdivision and semitotal point graphs of Archimedean lattices on some toplogical indices. volume 22, pages 830-837. Proceedings of the Jangieon Mathematical society, 2019.

[3] J. Devillers and A. T. Balaban (Eds.). Gordon and Breach, Amsterdam, 1999.

[4] Budak F.U and Beyli E.D. Detection of resistivity for antibiotics by probabilistic neural networks. Journal of Medical Systems, 35:87-91, 2011.

[5] C. K. Gupta, V. Lokesha, and S. B. Shetty. On the Symmetric division deg index of graph. 41(1):59-80, 2016.

[6] C. K. Gupta, V. Lokesha, S. B. Shetty, and P. S. Ranjini. Graph Operations on Symmetric Division Deg Index of Graphs. 6(1):280286, 2017.

[7] M. Javaid and Jinde Cao. Computing topological indices of probabilistic neural network. 30:38693876, 2018.

[8] V. Lokesha and T. Deepika. Symmetric division deg index of tricyclic and tetracyclic graphs. volume 7, pages 53-55. International Journal of Scientific and Engineering Research, 2016.

[9] V. Lokesha, T. Deepika, P. S. Ranjini, and Cangul. I. N., Operations of nanostructures via $S D D, A B C_{4}, G A_{5}$ indices. 2(1):173-180, 2017.

[10] V. Lokesha, M. Manjunath, and K.M. Devendraiah. Adriatic indices and Sanskruti index envisage of carbon nanocone. 9(4):280286, 2019.

[11] Bascil MS and Oztekin H. A study on hepatitis disease diagnosis using probabilistic neural network. 36:1603-1606, 2012. 
[12] Damir Vukicevic and Marija Gasperov. Bond additive modeling 1. Adriatic Indices. $83(3), 2010$.

[13] Zeba Y, B. Chaluvaraja, V. Lokesha, and Deepika T. Computation of Misbalanced type degree indices of certain classes of derived regular graph. 3:6169, 2019.

[14] Zeba Y, V. Lokesha, and Deepika. T. Graph Operations on Symmetric Division Deg Index of Graphs. 28(1):69-76, 2019. 